\title{
A technology for memory
}

Two methodological approaches allow researchers to manipulate the formation and reactivation of memories in mice.

When the narrator in Marcel Proust's novel dipped his madeleine in tea that singular morning and then tasted it, his brain instantaneously transported him through a stream of childhood memories.

Proust's character's recollection was triggered by a sensory perception - the taste of the madeleine-in a process called contextdependent memory.

Susumu Tonegawa, at the Massachusetts Institute of Technology, is one of several scientists interested in how the brain of mammals stores and retrieves these types of memories. It is thought, he explains, that when we experience something, this experience alters the physical or chemical nature of subpopulations of neurons, and these cells become activated. Later on, when we perceive a cue (a particular sight, smell or taste) linked to that experience, the same ensemble of neurons will be reactivated, producing the recollection of the entire memory.

But proving the cellular basis of memory encoding and recall has not been easy. It requires ways to identify the exact cells that become activated during the encoding of a given experience and means to specifically reactivate them subsequently in the context of a testable behavioral paradigm. Researchers from the labs of Tonegawa and of Mark Mayford, at the Scripps Research Institute, recently described methodological strategies to achieve just that.

To label cells that become activated during the encoding of a memory, the two groups used transgenic mice expressing the transcriptional activator tTA, under the control of the promoter of the immediate early gene Fos-a marker of recent neuronal activation. All it took then was to place a gene coding for a protein that can activate cells on cue, under tTA regulation. This genetic cascade is ultimately controlled by the addition of doxycycline, ensuring that only cells that are active during a given time window will be

susceptible to experimentally induced activation at a later time.

To study the mechanisms of memory formation and retrieval in mice, both groups applied this genetic approach in combination with a memorylearning paradigm called contextual fear conditioning. But they had different aims.

Tonegawa and his group asked a simple yet crucial question: "We wanted to test the

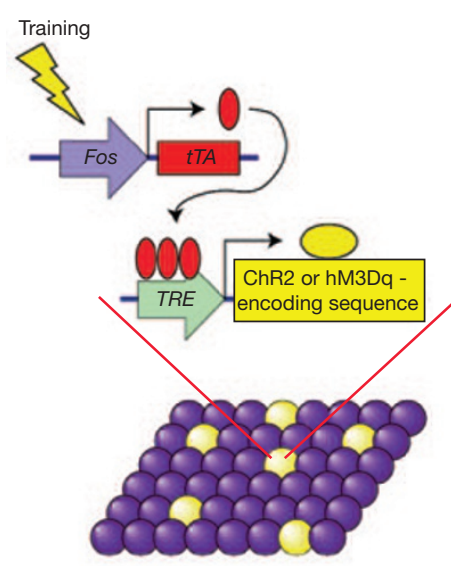

Genetic strategy used to tag active cells during a memory-learning paradigm. Adapted from Nature.
They then used this system to activate neurons associated with 'context $A$ ' while the mice were encoding a memory associated with 'context $B$ '. Under these conditions, the mice created a composite memory that integrated the artificially generated experience (the activity of those cells associated with context $A$ ) as well as the real one (the activity of the cells endogenously activated by context $B$ ). hypothesis that the reactivation of a small and specific set of cells involved in the encoding of the fear memory is sufficient to induce memory recall," he says. They targeted the expression of the gene that codes for the light-sensitive cation channel channelrhodopsin 2 (ChR2) under Fos promoter and tTA control to a hippocampal region called the dentate gyrus. Reactivation of the same cells that had become active during learning of the memory with light enabled them to test the sufficiency of this neuronal ensemble for context-dependent memory retrieval.

When the group saw the mice freeze in response to the activation of the targeted set of cells, in a context that otherwise had not been associated with fear, they knew that their hypothesis was right (Liu et al., 2012).

In contrast, Mayford and his group wanted to test whether the brain can integrate an artificially induced memory into the encoding of a natural one.

Instead of ChR2, Mayford's team targeted the expression of a synthetic $\mathrm{G}$ protein-coupled receptor (called $\mathrm{hM}_{3} \mathrm{D}_{\mathrm{q}}$ ) under similar genetic control. This receptor is activated by a small molecule, which the group administered systemically to target neurons distributed throughout the whole brain (Garner et al., 2012).
Mayford thinks that these studies can teach us something about how the brain integrates information from different sources to build contextual memories "when we learn new things we don't learn everything de novo, separated from everything else, we put information into a schema and that old information might also be represented in the brain."

Whether the findings of these two studies apply beyond this particular type of memory paradigm is an open question. It will be interesting to see whether the brain uses similar principles to remember long-gone events, such as those evoked by the taste of the madelaine in Proust's novel.

Tonegawa has an even greater challenge in mind for this technology. "If we can sort out the differences between the brain right before it remembers and after it has remembered, among those differences we should find some of the mechanisms underlying awareness," he says, "that's the dream I have."

\section{Erika Pastrana}

\section{RESEARCH PAPERS}

Garner A.R. et al. Generation of a synthetic memory trace. Science 335, 1513-1516 (2012).

Liu X. et al. Optogenetic stimulation of a hippocampal engram activates fear memory recall. Nature advance online publication (22 March 2012). 